\title{
Freie Universität
}

Title: Hydration Repulsion Difference between Ordered and Disordered Membranes Due to Cancellation of Membrane-Membrane and Water-Mediated Interactions

Author(s): Kowalik, B., Schlaich, A., Kanduč, M., Schneck, E., \& Netz, R. R.

Document type: Postprint

Terms of Use: $\quad$ Copyright applies. A non-exclusive, non-transferable and limited right to use is granted. This document is intended solely for personal, non-commercial use.

Citation:

Kowalik, B., Schlaich, A., Kanduč, M., Schneck, E., \& Netz, R. R. (2017). Hydration Repulsion Difference between Ordered and Disordered Membranes Due to Cancellation of MembraneMembrane and Water-Mediated Interactions. The Journal of Physical Chemistry Letters, 8(13), 2869-2874. https://doi.org/10.1021/acs.jpclett.7b00977

This document is the Accepted Manuscript version of a Published Work that appeared in final form in The Journal of Physical Chemistry Letters, copyright @ American Chemical Society after peer review and technical editing by the publisher. To access the final edited and published work see https://doi.org/10.1021/acs.jpclett.7b00977. 


\title{
Hydration repulsion difference between ordered and disordered membranes due to cancellation of membrane-membrane and water-mediated interactions
}

\author{
Bartosz Kowalik, ${ }^{1}$ Alexander Schlaich, ${ }^{1}$ Matej Kanduč, ${ }^{2}$ Emanuel Schneck, ${ }^{3}$ and Roland R. Netz ${ }^{1}$ \\ ${ }^{1}$ Department of Physics, Freie Universität Berlin, 14195 Berlin, Germany \\ ${ }^{2}$ Institut für Weiche Materie und Funktionale Materialien, \\ Helmholtz-Zentrum Berlin, 14109 Berlin, Germany \\ ${ }^{3}$ Biomaterials Department, Max Planck Institute of Colloids and Interfaces, 14476 Potsdam, Germany
}

(Dated: March 13, 2017)

Hydration repulsion acts between all sufficiently polar surfaces in water at small separations and prevents dry adhesion up to kilobar pressures [1]. Yet it remained unclear whether this ubiquitous force depends on surface structure or is a sole water property [2]. We demonstrate that previous deviations among different experimental measurements of pressures in phospholipid bilayer stacks disappear when plotting data consistently as a function of repeat distance or membrane surface distance. The resulting pressure versus distance curves agree quantitatively with our atomistic simulation results and exhibit different decay lengths in the ordered gel and the disordered fluid states. This suggests that hydration forces are not caused by water ordering effects alone. Splitting the simulated total pressure into membrane-membrane and water-mediated parts shows that these contributions are opposite in sign and of similar magnitude, they thus are equally important. The resulting net hydration pressure between membranes is what remains from the near-cancellation of these ambivalent contributions.

Even electrically neutral polar surfaces repel in water and exhibit for small separations, when the last water layers are removed, an exponential repulsive force that is commonly called hydration force. The mechanism behind this force and even its name are intensely debated [2]; what is generally acknowledged, however, is that it is ubiquitous and acts between self-assembled membranes and surfactant layers [3], colloids [4], clays and biomolecules such as DNA [5] and proteins [6]. Hydration forces are thus important for diverse processes such as membrane fusion and adhesion [7], soap bubble stability, protein adsorption [8] as well as lubrication of biological [9] and synthetic materials [10].

Different concepts were invoked to rationalize hydration forces. As early discussed by Langmuir [11], an effective surface repulsion was suggested to arise from the removal of strongly bound hydration layers, hence the name hydration force (to which we stick for historic reasons without reference to the implied mechanism). The overlap of water ordering profiles at two opposing surface was theoretically shown to produce an exponentially decaying repulsion $[12,13]$ and reasoned to explain the universality of hydration forces observed for different surfaces [1]. On the other hand, the presence of oscillatory forces between stiff surfaces measured with the surface-force apparatus [14], and in particular the huge spectrum of observed hydration force amplitudes and decay lengths for different surfaces, was used to argue that additional, direct surface interactions (encompassing entropic effects due to the perturbation of conformational surface degrees of freedom) must play an equally important role for small surface separations [15].

Historically, experiments on lipid bilayers for several reasons played a pivotal role: For given lipid chemistry and temperature, and in the absence of cosolutes, the self-assembled bilayer structure uniquely depends on a single parameter, namely the mixing ratio of water and lipids, thereby excluding ambiguities related to different preparations or compositions as for most solid surfaces. Besides, for neutral lipids there is no need to subtract the electrostatic double-layer repulsion, a procedure which adds significant arbitrariness to the definition of the hydration force for charged surfaces [16]. In addition, osmotic stress techniques allow to measure the repeat distance in a multilamellar stack as a function of the imposed osmotic pressure with high precision and over a vast range of pressures [17]. Finally, the presence of many surfaces dilutes contaminations and increases accuracy due to the parallel detection of multiple repeat distances in one measurement. As a matter of fact, supported bilayers exhibit similar hydration forces as free bilayer stacks [18], demonstrating that undulation forces (which are suppressed for supported bilayers) are 
negligible for small bilayer separations.

However, the key experiment on phospholipid bilayers led to puzzling results, which severely hampers the complete understanding of hydration forces. Phospholipid membranes display a main transition from an ordered gel-like state at low temperature to a disordered fluid state at high temperature, which is well studied due to its physiological relevance [1921]. The chemical surface composition does not change during this transition, only the surface structure; the comparison of hydration forces in the gel and fluid states is thus of paramount importance since it should allow to decide whether direct surface interactions or water ordering, the latter presumably being similar in the gel and fluid states, are the dominating contributor to hydration forces. The first experimental study indeed yielded different hydration force curves as a function of surface separation in the gel and fluid states, suggesting that hydration forces are not solely caused by water effects [22]. However, later experiments with the same phospholipid gave dissonant results [23-25]. It was early on suggested that this comes from different definitions of the interface position between water and bilayers used in the analysis of the experimental data [26], but this was never settled.

In this paper we first demonstrate that all five published experimental hydration pressure curves for Dipalmitoylphosphatidylcholine (DPPC), some of which in the gel and some in the fluid state, are consistent when plotted as a function of the bilayer repeat distance $D$, which is the primary quantity measured in scattering experiments. For this we undo the conversion of experimentally measured repeat distances $D$ to reported surface separations, for which different definitions have been used. We then convert $D$ to the water slab thickness $D_{\text {w }}$ using the thermodynamic definition of the Gibbs dividing surface. We next show that the experimental data quantitatively agree with simulations of DPPC bilayers performed at low temperature in the gel state and at high temperature in the fluid state when plotted as a function of $D_{\mathrm{w}}$. This comparison reveals that the decay lengths are vastly different in the gel and fluid states, hinting that hydration forces are not solely caused by water ordering effects. Finally, and most importantly, further analysis of the simulation results shows that the total interaction pressure results from the near cancellation of attractive direct membrane-membrane interaction and repulsive indirect interaction, the latter being comprised of water-water and water-membrane interactions. $\mathrm{Cu}-$ riously, direct and indirect interactions have almost the same magnitude, both in the gel and the fluid states, and for separations $D_{\mathrm{w}}>1 \mathrm{~nm}$ exhibit similar exponential decay lengths of about $\lambda \approx 0.2 \mathrm{~nm}$. The sum of direct and indirect forces, which together make up what is called the hydration force, is smaller than both direct and indirect force by a factor of roughly ten. The hydration force decay length turns out to be $\lambda_{\text {gel }} \approx 0.2 \mathrm{~nm}$ in the gel and $\lambda_{\text {fluid }} \approx 0.4 \mathrm{~nm}$ in the fluid state. Thus, the hydration force cannot be explained by water-ordering or direct surfacesurface interactions alone, simply because it is the sum of these two competing contributions of almost equal magnitude. Due to the near-cancellation of the direct and indirect contributions, the resulting hydration force depends on fine details of both contributions in a very subtle manner. It comes at no surprise that the hydration force behaves very differently from these contributions, both in terms of its amplitude but also in terms of its range (i.e. its exponential decay length). This should be kept in mind when trying to explain hydration forces in terms of simple theoretical concepts (which typically consider only one part of the problem) and is vividly demonstrated by the deviating hydration forces in the gel and fluid states.

In Fig. 1 (a) we reproduce all available experimental data [22-25] for the osmotic pressure $p$ of DPPC multilamellar stacks as a function of the published water slab thickness $D_{\text {w }}$ in a $\log$-lin representation, two data sets correspond to the gel state (squares) and three to the fluid state (crosses). Most strikingly, different data sets in the gel and fluid states disagree among each other, as was noted before [27], and give rise to significantly different decay lengths $\lambda_{\text {gel }}=0.11 \mathrm{~nm}, 0.18 \mathrm{~nm}$ and $\lambda_{\text {fluid }}=0.18 \mathrm{~nm}$, $0.22 \mathrm{~nm}, 0.26 \mathrm{~nm}$ (indicated by straight lines, see Table S1 in SI) as extracted from fits to $p=p_{0} e^{-D_{\mathrm{w}} / \lambda}$. Thus, while the decay lengths in the gel and fluid states differ, the inconsistencies among different experiments preclude any interpretation of these results.

In fact, different experiments used different methods to convert the experimentally measured lamellar repeat distance $D$, which is the sum of the water slab thickness $D_{\mathrm{w}}$ and the lipid membrane thickness $D_{1}$, to the water slab thickness $D_{\mathrm{w}}$ : In one method, $D_{\mathrm{w}}$ is derived from the known lipid-water mixing ratio and assuming water and lipids to be incompressible [22]. In a different treatment the lipid membrane thickness $D_{1}$ is determined from electron density profiles derived from X-ray diffraction and from that $D_{\mathrm{w}}=D-D_{\mathrm{l}}$ is computed. In the third treatment $D_{\mathrm{w}}$ follows from the bilayer area compressibility on the basis of $D_{1}$ at one reference pressure [24, 25] (see SI for details on the different conversion methods). 

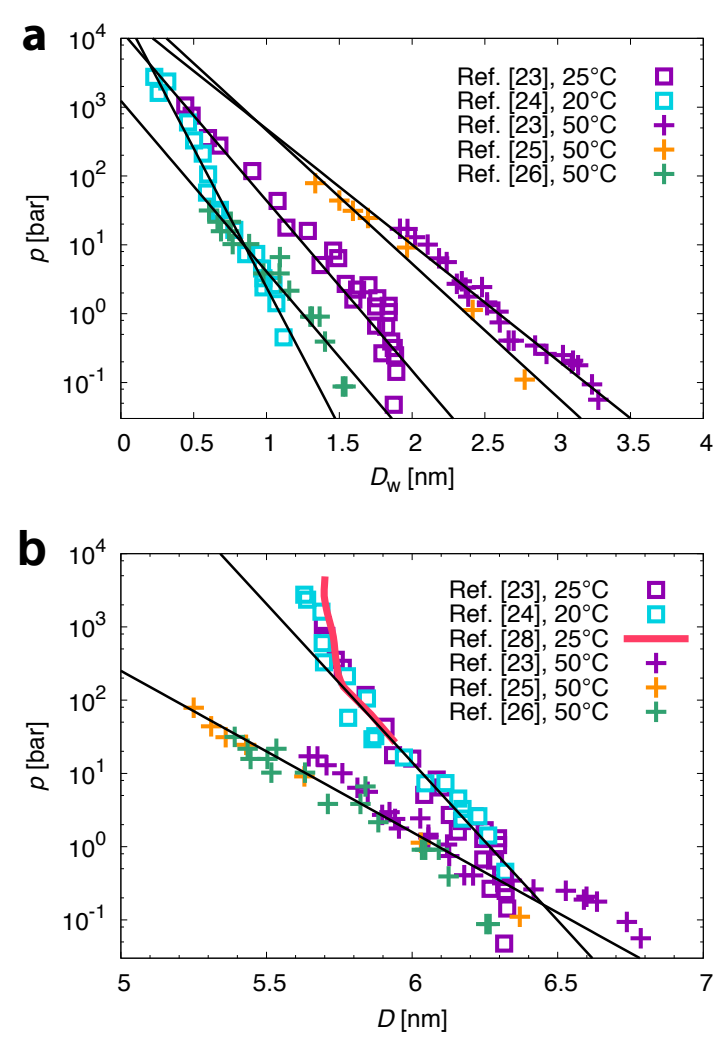

FIG. 1. Experimental osmotic pressure data for DPPC multilamellar stacks. a, Pressure data for DPPC in the gel (squares) and fluid (crosses) states as a function of the reported water slab thickness $D_{\mathrm{w}}$. Black lines indicate exponential fits. $\mathbf{b}$, Pressures as a function of the reconstructed lamellar repeat distance $D$. Black lines represent exponentials with decay lengths $\lambda_{\text {fluid }}=$ $0.20 \mathrm{~nm}$ and $\lambda_{\text {gel }}=0.10 \mathrm{~nm}$.

In Fig. 1 (b) we present the same experimental pressure data as a function of the lamellar repeat distance $D$. Note that in one case the $p(D)$ data was not given [22], so we converted the data from $D_{\mathrm{w}}$ to $D$. We also include microcalorimetry data [28] that reports the osmotic pressure $p$ as a function of the water-lipid ratio. Excellent agreement between all available experimental data is observed, which endorses that multilamellar systems constitute exceptionally robust experimental systems. We conclude that deviations between experiments in Fig. 1 (a) are indeed caused by different conversion methods used to derive $D_{\mathrm{w}}$ from the experimentally measured repeat distance $D$. Clearly, Fig. 1 (b) suggests an exponential pressure decay versus $D$ with decay lengths that are very different in the gel and fluid states, $\lambda_{\text {fluid }}=0.20 \mathrm{~nm}$ and $\lambda_{\text {gel }}=0.10 \mathrm{~nm}$ (indi- cated by black lines). However, the membrane thickness $D_{1}$ depends sensitively on pressure, reflected by the fact that the relation between $D$ and $D_{\text {w }}$ is highly non-linear (see SI). The function $p(D)$ in Fig. 1 (b) includes hydration force and membrane compression effects, only the function $p\left(D_{\mathrm{w}}\right)$ corresponds to the hydration force per se, which thus requires careful definition of $D_{\mathrm{w}}$.

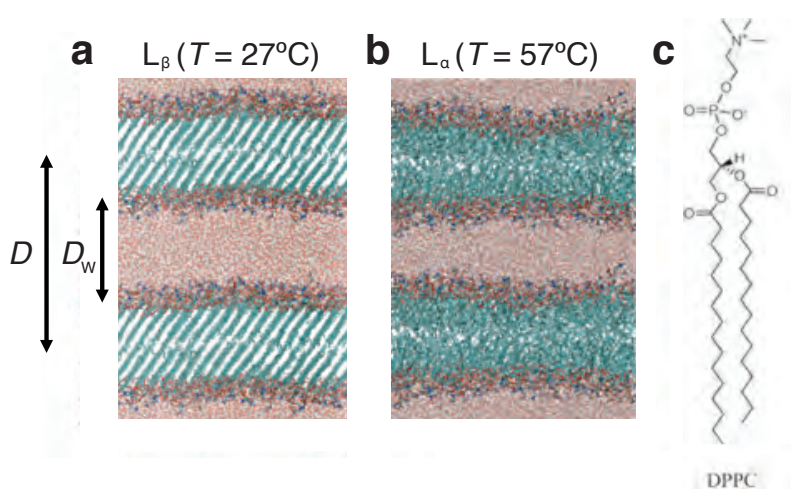

FIG. 2. Simulation setup. a,b, Simulation snapshots of a DPPC lipid bilayer in the (a) gel $L_{\beta}$ phase and in the (b) fluid $L_{\alpha}$ phase. The simulation box contains one periodically replicated hydrated bilayer, which for clarity is duplicated in the $z$ direction. c, Chemical structure of a DPPC lipid.

Molecular dynamics (MD) simulations allow to model hydrated bilayers in atomistic detail [29]. Simulations at controlled water chemical potential $[30,31]$ are possible by the thermodynamic extrapolation method, which allows to quantitatively compare experimental and simulated interaction pressures [32]. Snapshots of our simulations in fluid and gel states together with the DPPC chemical structure are presented in Fig. 2.

In Fig. 3 we compare the interaction pressure from simulations in the osmotic pressure ensemble at fixed hydrostatic pressure of 1 bar (triangles) with experimental data (squares and crosses) in a) gel and b) fluid states as a function of the water slab thickness $D_{\text {w }}$. We calculate $D_{\text {w }}$ based on the Gibbs-diving surface position, which amounts to $D_{\mathrm{w}}=N_{\mathrm{w}} v_{\mathrm{w}}^{0} / A$ where $N_{\mathrm{w}}$ is the number of water molecules in one layer, $v_{\mathrm{w}}^{0}$ is the volume per water molecule in bulk, and $A$ is the system area. Incidentally, this is the same conversion used in one experiment [22] and is based on the unambiguous thermodynamic definition of the membrane-water interface position. We use the same conversion for all experimental data sets. For both gel and fluid states, we observe excel- 

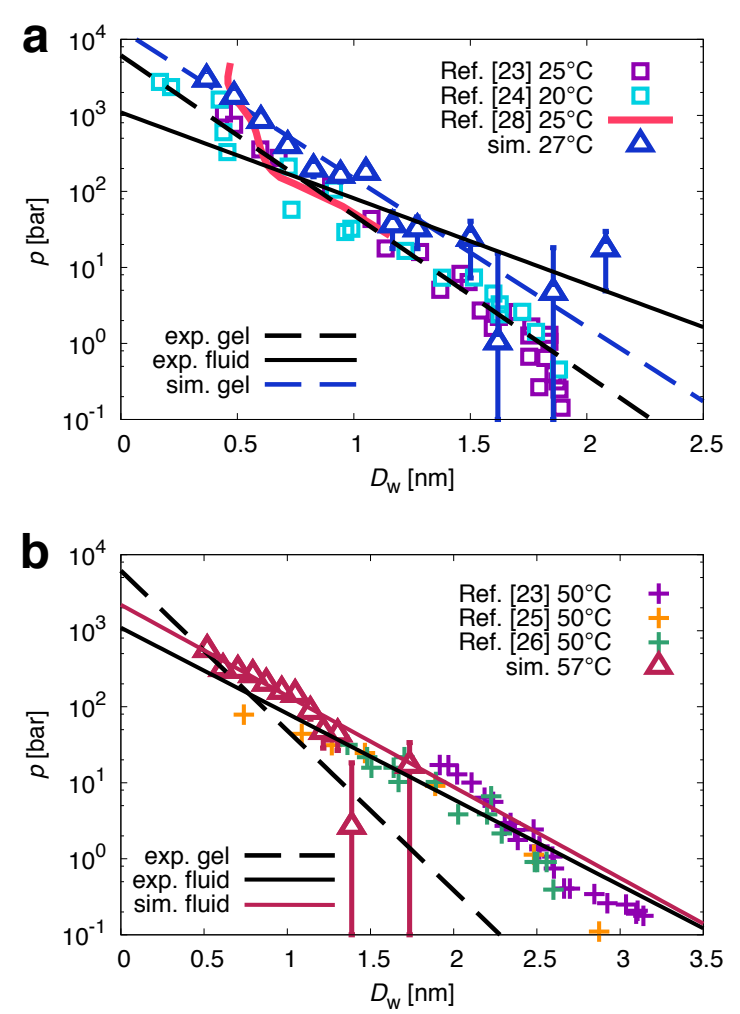

FIG. 3. Osmotic pressure data with consistent definition of the water slab thickness. a,b, Comparison of bilayer pressure from simulations (triangles) and experiments (squares and crosses) as function of water slab thickness $D_{\mathrm{w}}$ in (a) gel and (b) fluid state. Exponential fits to the experimental data give decay lengths $\lambda_{\text {gel }}=0.21 \pm 0.01 \mathrm{~nm}$ (black broken line) for a fit range $[0$, $1.8 \mathrm{~nm}]$ and $\lambda_{\text {fluid }}=0.38 \pm 0.02 \mathrm{~nm}$ (black solid line) for a fit range $[0,2.6 \mathrm{~nm}]$. Fits to the simulation data yield decay lengths $\lambda_{\text {gel }}=0.22 \pm 0.02 \mathrm{~nm}$ (blue broken line) and $\lambda_{\text {fluid }}=0.36 \pm 0.02 \mathrm{~nm}$ (red solid line) for fit ranges $[0,1.3 \mathrm{~nm}]$ and $[0,1.4 \mathrm{~nm}]$, respectively, restricted to the distance range where pressures are strictly positive.

lent agreement among experimental and simulated pressure curves. The experimental decay length in the gel state is $\lambda_{\text {gel }}=0.21 \mathrm{~nm}$, in the fluid state we obtain $\lambda_{\text {fluid }}=0.38 \mathrm{~nm}$. Thus the experimental decay lengths for $p\left(D_{\mathrm{w}}\right)$ differ among fluid and gel states and at the same time deviate significantly from the decay lengths of $p(D)$ in Fig. 1 (b). This clearly rules out a pure water-mediated mechanism for the hydration repulsion, because in this case not the decay length but only the hydration force amplitude should differ in the gel and fluid states. Fits to the simulation data yield $\lambda_{\text {gel }}=0.22 \mathrm{~nm}$ and $\lambda_{\text {fluid }}=0.36 \mathrm{~nm}$, hence in good agreement with the experiments. This validates our further simulation analysis.

To gain insight into the origin of the hydration force, we decompose the total pressure $p$ into the direct $p_{\text {dir }}$ and indirect parts $p_{\text {ind }}[32]$, where $p_{\text {dir }}$ contains all membrane-membrane interactions, whereas $p_{\text {ind }}=p-p_{\text {dir }}$ contains the remaining water-water and water-membrane forces. This decomposition is only possible in the hydrostatic ensemble at fixed water chemical potential (see SI Sec. 8). Note that this splitting is position independent and thus adds minimal ambiguity.

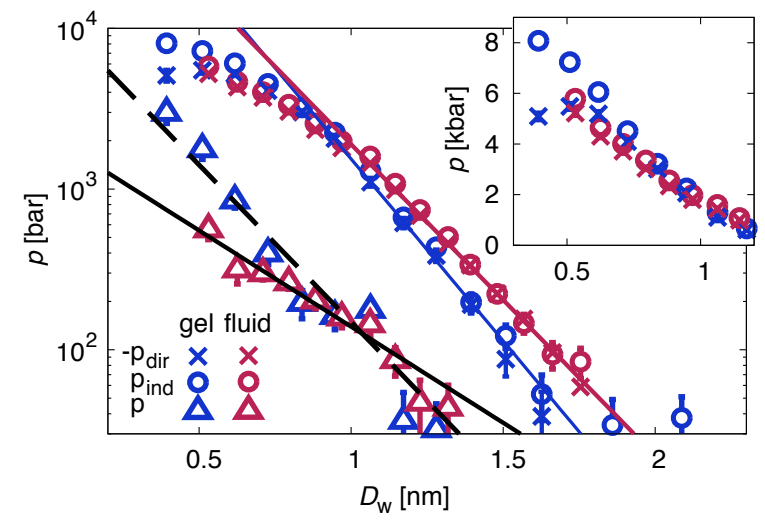

FIG. 4. Pressure decomposition. Decomposition of the simulated pressure (triangles) into direct interactions between DPPC membranes $-p_{\text {dir }}$ (crosses) and the indirect contribution $p_{\text {ind }}=p-p_{\text {dir }}$ (circles) in the gel (blue) and fluid states (red). Colored lines are simultaneous exponential fits to the direct and indirect contributions for $D_{\mathrm{w}}>1 \mathrm{~nm}$ with decay lengths $\lambda_{\text {gel }}=0.19 \mathrm{~nm}$ in the gel (blue line) and $\lambda_{\text {fluid }}=0.22 \mathrm{~nm}$ in the fluid state (red line). The exponential fits to the total pressures from Fig. 3 are included as black lines. In the inset $-p_{\text {dir }}$ and $p_{\text {ind }}$ are shown on a linear scale.

In Fig. 4 we plot the total pressure $p$ together with the indirect pressure $p_{\text {ind }}$ and (since it is attractive) the negative direct pressure $-p_{\text {dir }}$ in the gel and fluid states. We observe that $-p_{\text {dir }}$ and $p_{\text {ind }}$ are very similar to each other and thus nearly cancel, consequently, the total pressure $p=p_{\text {ind }}+p_{\text {dir }}$ is much reduced and smaller by roughly an order of magnitude. This holds for both gel and fluid data. The direct and indirect pressures exhibit for separations $D_{\mathrm{w}}>1 \mathrm{~nm}$ an exponential decay which is characterized by surprisingly similar decay lengths, roughly given by $\lambda_{\text {gel }}=0.19 \mathrm{~nm}$ in the gel state and $\lambda_{\text {fluid }}=0.22 \mathrm{~nm}$ in the fluid state (indicated by blue 
and red solid lines). The sum of these contributions, the total pressure $p$, however shows different exponential decay lengths of $\lambda_{\text {gel }}=0.22 \mathrm{~nm}$ in the gel and $\lambda_{\text {fluid }}=0.36 \mathrm{~nm}$ in the fluid states, as already shown and discussed in Fig. 3. The significant difference between the gel and fluid total pressures is thus caused by relatively tiny differences in the direct and indirect contributions, which only survive since $p_{\text {dir }}$ and $p_{\text {ind }}$ almost exactly cancel. To look into this, we plot $-p_{\text {dir }}$ and $p_{\text {ind }}$ in a lin-lin representation in the inset of Fig. 4 . There it is seen that $-p_{\text {dir }}$ in the fluid and gel states are very similar to each other, while the indirect (water-mediated) contributions $p_{\text {ind }}$ differ substantially for small separations. We conclude that the difference between the total pressures $p$ in fluid and gel states is mainly caused by a relatively small difference in the watermediated indirect contribution $p_{\text {ind }}$. The different decay lengths of the total pressure $p$ in fluid and gel states comes as a surprise; given the similarity of direct and indirect contributions, it transpires why an understanding of the hydration force could not be gained from consideration of the water-mediated or the direct membrane--membrane interactions alone, which has been the prevalent mode of thinking in the literature so far.
[1] V. Parsegian and T. Zemb, Current opinion in colloid \& interface science 16, 618 (2011).

[2] J. N. Israelachvili and H. Wennerstroem, The Journal of Physical Chemistry 96, 520 (1992).

[3] R. Rand and V. Parsegian, Biochimica et Biophysica Acta (BBA) - Reviews on Biomembranes 988, 351 (1989).

[4] W. A. Ducker, T. J. Senden, and R. M. Pashley, Nature 353, 239 (1991).

[5] D. Rau, B. Lee, and V. Parsegian, Proceedings of the National Academy of Sciences 81, 2621 (1984).

[6] S. Leikin, D. Rau, and V. Parsegian, Proceedings of the National Academy of Sciences 91, 276 (1994).

[7] S. L. Leikin, M. M. Kozlov, L. V. Chernomordik, V. S. Markin, and Y. A. Chizmadzhev, Journal of Theoretical Biology 129, 411 (1987).

[8] D. R. Lu, S. J. Lee, and K. Park, Journal of Biomaterials Science, Polymer Edition 3, 127 (1992).

[9] A. Gaisinskaya, L. Ma, G. Silbert, R. Sorkin, O. Tairy, R. Goldberg, N. Kampf, and J. Klein, Faraday Discuss. 156, 217 (2012).

[10] H. Chen, J. R. Cox, H. Ow, R. Shi, and A. Z. Panagiotopoulos, Scientific Reports 6 (2016).

[11] I. Langmuir, The Journal of Chemical Physics 6, 873 (1938).

[12] S. Marčelja and N. Radić, Chemical Physics Letters 42, 129 (1976).

[13] G. Cevc, R. Podgornik, and B. Žekš, Chemical Physics Letters 91, 193 (1982).

[14] J. Israelachvili and P. McGuiggan, Science (New York, N.Y.) 241, 795-800 (1988).

[15] J. Israelachvili and H. Wennerström, Nature 379, $219-225$ (1996).

[16] R. Pashley, Journal of Colloid and Interface Science 80, 153 (1981).

[17] D. M. LeNeveu, R. P. Rand, and V. A. Parsegian, Nature 259, 601 (1976).

[18] R. Horn, J. Israelachvili, J. Marra, V. Parsegian, and R. Rand, Biophysical journal 54, 1185 (1988).

[19] H. Ichimori, T. Hata, T. Yoshioka, H. Matsuki, and
S. Kaneshina, Chemistry and Physics of Lipids 89, 97 (1997).

[20] M. Edidin, Annual review of biophysics and biomolecular structure 32, 257 (2003).

[21] G. Van Meer, D. R. Voelker, and G. W. Feigenson, Nature reviews Molecular cell biology 9, 112 (2008).

[22] L. Lis, M. McAlister, N. Fuller, R. Rand, and V. Parsegian, Biophysical journal 37, 657 (1982).

[23] T. J. McIntosh and S. A. Simon, Biochemistry 32, 8374 (1993).

[24] K. Gawrisch, D. Ruston, J. Zimmerberg, V. Parsegian, R. Rand, and N. Fuller, Biophysical Journal 61, 1213 (1992).

[25] H. I. Petrache, N. Gouliaev, S. Tristram-Nagle, R. Zhang, R. M. Suter, and J. F. Nagle, Physical Review E 57, 7014 (1998).

[26] T. J. McIntosh, Current Opinion in Structural Biology 10, 481 (2000).

[27] T. J. McIntosh and S. A. Simon, Biochemistry 25, 4058 (1986)

[28] N. Markova, E. Sparr, L. Wadsö, and H. Wennerström, The Journal of Physical Chemistry B 104, 8053 (2000).

[29] D. Tieleman, S. Marrink, and H. Berendsen, Biochimica et Biophysica Acta (BBA) - Reviews on Biomembranes 1331, 235 (1997).

[30] L. Hua, R. Zangi, and B. J. Berne, The Journal of Physical Chemistry C 113, 5244 (2009).

[31] T. Hayashi, A. J. Pertsin, and M. Grunze, The Journal of Chemical Physics 117, 6271 (2002).

[32] E. Schneck, F. Sedlmeier, and R. R. Netz, Proceedings of the National Academy of Sciences 109, 14405 (2012).

[33] B. Hess, C. Kutzner, D. van der Spoel, and E. Lindahl, J. Chem. Theory Comput. 4, 435 (2008).

[34] D. Tieleman and H. J. C. Berendsen, J. Chem. Phys. 105, 4871 (1996).

[35] O. Berger, O. Edholm, and F. Jahnig, Biophys. J. 72, 2002 (1997).

[36] E. Lindahl and O. Edholm, Biophys. J. 79, 426 
(2000).

[37] H. J. C. Berendsen, J. R. Grigera, and T. P. Straatsma, J. Phys. Chem. 91, 6269 (1987).

[38] T. Schubert, E. Schneck, and M. Tanaka, The Journal of Chemical Physics 135, 055105 (2011).

[39] G. Bussi, D. Donadio, and M. Parrinello, The Journal of Chemical Physics 126, 014101 (2007).

[40] B. Kowalik, T. Schubert, H. Wada, M. Tanaka, R. R. Netz, and E. Schneck, The Journal of Physical Chemistry B 119, 14157 (2015).

[41] P. S. Coppock and J. T. Kindt, The Journal of Physical Chemistry B 114, 11468 (2010).

[42] J. Ulmius, H. Wennerström, G. Lindblom, and G. Arvidson, Biochemistry 16, 5742 (1977).

[43] C. Grabielle-Madelmont and R. Perron, Journal of colloid and interface science 95, 471 (1983).

[44] H. J. C. Berendsen, J. P. M. Postma, W. F. van Gunsteren, A. DiNola, and J. R. Haak, J. Chem. Phys. 81, 3684 (1984).

[45] T. Darden, D. York, and L. Pedersen, J. Chem. Phys. 98, 10089 (1993).

[46] U. Essmann, L. Perera, and M. L. Berkowitz, Langmuir 11, 4519 (1995).

[47] B. Widom, J. Chem. Phys 39, 2808 (1963).

[48] M. R. Shirts and J. D. Chodera, The Journal of Chemical Physics 129, 124105 (2008).

\section{METHODS}

We use the Gromacs simulation package [33] with the Berger lipid force field [34-36] and the SPC/E water model [37]. The assisted freezing method [38] is used for the construction of fully hydrated membranes in the $L_{\beta}$ (gel) phase (Fig. 2 a) at a temperature of $T=270 \mathrm{~K}$, controlled by the v-rescale thermostat [39]. This fully hydrated membrane consists of $2 \times 36$ DPPC lipids hydrated by 40 water molecules per lipid. The structure is equilibrated at $T=300 \mathrm{~K}$ and afterwards gradually dehydrated by one molecule per lipid and each time equilibrated for 5 ns down to a hydration level of 3 waters per lipid molecule. All equilibrations are performed in the NpT ensemble. To improve sampling, we use four different starting configurations, which are independently dehydrated five times with different random seeds, giving 20 different systems per hydration level. For production runs in the $L_{\alpha}$ fluid phase (Fig. 2 b) the temperature in the gel state is increased to 330 $\mathrm{K}$, above the melting temperature of DPPC membranes in experiments and in simulations [40-43].

All simulations are performed with periodic boundary conditions and a time step of $2 \mathrm{fs}$. An anisotropic pressure coupling is employed using the Berendsen barostat [44] with a time constant of $\tau_{\mathrm{P}}=2 \mathrm{ps}$. Lennard-Jones interactions are cut-off at $0.9 \mathrm{~nm}$ and electrostatics use the Particle-MeshEwald (PME) method $[45,46]$ with a $0.9 \mathrm{~nm}$ realspace cutoff. Each system is simulated for $5 \mathrm{~ns}$, so the total simulation time is $100 \mathrm{~ns}$ per hydration level. In the osmotic ensemble, the pressure is set to $p=1$ bar and chemical potential $\mu$ is measured using the Test Particle Insertion method [47] for the van-der-Waals contribution and the thermodynamic integration for the electrostatic contribution making use of the Multistate Bennett Acceptance Ratio (MBAR) method [48]. From the 20 different systems per hydration level the error of the chemical potential and thus the osmotic pressure is estimated.

In the MD simulations, the water slab thickness $D_{\mathrm{w}}$ is defined by $D_{\mathrm{w}}=N v_{\mathrm{w}} / A$, where $N$ is the number of water molecules in the system, $v_{\mathrm{w}}$ is the volume of one water molecule and $A$ is the simulation box area. We measured $\rho_{\text {bulk }}(T=300 \mathrm{~K})=$ $985 \mathrm{~kg} / \mathrm{m}^{3}$ and $\rho_{\text {bulk }}(T=330 \mathrm{~K})=967 \mathrm{~kg} / \mathrm{m}^{3}$ in water bulk simulations, which correspond to $v_{\mathrm{w}}=$ $0.0304 \mathrm{~nm}^{3}$ for $T=300 \mathrm{~K}$ and $v_{\mathrm{w}}=0.0309 \mathrm{~nm}^{3}$ for $T=330 \mathrm{~K}$. With this definition, the water slab thickness equals the distance between the Gibbs dividing surfaces that are located on both sides of the water slab.

Using the equation $\Delta \mu=-v_{\mathrm{w}}^{0} p$ (see SI for the derivation), the results for $\mu$ are converted into equivalent osmotic pressures. The hydrostatic simulations, used to decompose the pressure into direct and indirect parts, employ the predicted osmotic pressure, we explicitly checked that the resulting chemical potential equals the bulk water chemical potential. 